\title{
LOCAL INSTABILITY SIGNATURES IN ALMA OBSERVATIONS OF DENSE GAS IN NGC 7469
}

\author{
Kambiz Fathi ${ }^{1,2}$, Takuma Izumi ${ }^{3}$, Alessandro B. Romeo ${ }^{4}$, Sergio Martín ${ }^{5}$, Masatoshi Imanishi ${ }^{6,7,8}$, \\ Evanthia Hatziminaoglou ${ }^{9}$, Susanne Aalto ${ }^{4}$, Daniel Espada ${ }^{6,7,10}$, Kotaro Kohno ${ }^{3,11}$, Melanie Krips ${ }^{5}$, \\ Satoki Matsushita ${ }^{12}$, David S. Meier ${ }^{13}$, NaOmasa Nakai ${ }^{14}$, and Yuichi Terashima ${ }^{15}$ \\ ${ }^{1}$ Stockholm Observatory, Department of Astronomy, Stockholm University, SE-106 91 Stockholm, Sweden \\ ${ }^{2}$ Oskar Klein Centre for Cosmoparticle Physics, Stockholm University, SE-106 91 Stockholm, Sweden \\ ${ }^{3}$ Institute of Astronomy, The University of Tokyo, 2-21-1 Osawa, Mitaka, Tokyo 181-0015, Japan \\ ${ }^{4}$ Department of Earth and Space Sciences, Chalmers University of Technology, SE-41296 Gothenburg, Sweden \\ ${ }^{5}$ Institut de Radio Astronomie Millimétrique, Domaine Universitaire, 300 Rue de la Piscine, F-38406 Saint Martin d'Heres, France \\ ${ }^{6}$ National Astronomical Observatory of Japan, 2-21-1 Osawa, Mitaka, Tokyo 181-8588, Japan \\ ${ }^{7}$ The Graduate University for Advanced Studies (SOKENDAI), 2-21-1 Osawa, Mitaka, Tokyo 181-8588, Japan \\ ${ }_{9}^{8}$ Subaru Telescope, NAOJ, 650 North A'ohoku Place, Hilo, HI 96720, USA \\ ${ }^{9}$ ESO, Karl-Schwarzschild-Str. 2, D-85748 Garching bei München, Germany \\ ${ }^{10}$ Joint ALMA Observatory, Alonso de Cordova, 3107, Vitacura, Santiago 763-0355, Chile \\ ${ }^{11}$ Research Center for the Early universe, The University of Tokyo, 7-3-1 Hongo, Bunkyo, Tokyo 113-0033, Japan \\ ${ }_{12}$ Academia Sinica, Institute of Astronomy \& Astrophysics, P.O. Box 23-141, Taipei 10617, Taiwan \\ ${ }^{13}$ Department of Physics, New Mexico Institute of Mining and Technology, 801 Leroy Place, Soccoro, NM 87801, USA \\ ${ }_{14}$ Division of Physics, Faculty of Pure and Applied Sciences, Tsukuba, Ibaraki 305-8571, Japan \\ ${ }^{15}$ Department of Physics, Ehime University, 2-5 Bunkyo-cho, Matsuyama, Ehime 790-8577, Japan \\ Received 2015 March 25; accepted 2015 June 2; published 2015 June 19
}

\begin{abstract}
We present an unprecedented measurement of the disk stability and local instability scales in the luminous infrared Seyfert 1 host, NGC 7469, based on ALMA observations of dense gas tracers and with a synthesized beam of $165 \times 132$ pc. While we confirm that non-circular motions are not significant in redistributing the dense interstellar gas in this galaxy, we find compelling evidence that the dense gas is a suitable tracer for studying the origin of its intensely high-mass star-forming ringlike structure. Our derived disk stability parameter $\mathcal{Q}$ accounts for a thick disk structure, and its value falls below unity at the radii in which intense star formation is found. Furthermore, we derive the characteristic instability scale $\lambda_{c}$ and find a striking agreement between our measured scale of $\sim 180 \mathrm{pc}$ and the typical sizes of individual complexes of young and massive star clusters seen in high-resolution images.
\end{abstract}

Key words: galaxies: individual (NGC 7469) - galaxies: ISM - galaxies: kinematics and dynamics

\section{INTRODUCTION}

The central kiloparsec region of the barred Seyfert 1 host NGC 7469 displays a number of morphological and kinematic features that provide interesting clues about the dynamical behavior of the interstellar medium and its connection with star formation across the disk in this galaxy. Previous highresolution studies carried out by Meixner et al. (1990), Salamanca et al. (1995), Genzel et al. (1995), Davies et al. (2004, 2005), and Hicks et al. (2009) have shown that the gasrich central region displays intense star formation in a $R \sim 0.5-1 \mathrm{kpc}$ ringlike structure contributing to up to twothirds of the galaxy's bolometric luminosity $\left(L_{\text {bol }}=3 \times 10^{11} L_{\odot}\right)$. The ringlike structure is dominated by young $(<100 \mathrm{Myr})$ and massive $\left(10^{5}-10^{7} M_{\odot}\right)$ super star clusters, with an enclosed mass of $\sim 2.7 \times 10^{9} M_{\odot}$ (Mauder et al. 1994; Díaz-Santos et al. 2007). The individual clusters are grouped in $\sim 150-200 \mathrm{pc}$ scale star-forming complexes (DíazSantos et al. 2007). The relatively gas-poor nuclear 30-60 pc region hosts a $<100$ Myr nuclear star cluster that contributes to $10 \%$ of the nuclear bolometric luminosity and $30 \%$ of the nuclear $K$-band continuum light. Furthermore, the intensities of the cold and warm molecular gas are consistent with a CO-to$\mathrm{H}_{2}$ conversion factor similar to other star-forming galaxies, i.e., $X_{\mathrm{CO}}=0.4-0.8$ the conversion factor in the Milky Way (Davies et al. 2004; Bolatto et al. 2013).

The dynamics of the molecular gas around the nucleus is consistent with a thin disk model with $\sim 10^{8} M_{\odot}$ dynamical mass and $\sim 3100 M_{\odot} \mathrm{pc}^{-2}$ surface density (Hicks et al. 2009). The stellar structure inside the nuclear $30 \mathrm{pc}$ is dispersion dominated, whereas the $V / \sigma$ rapidly raises at this radius, staying consistently around unity out to $\sim 150 \mathrm{pc}$. Furthermore, Hicks \& Malkan (2008) applied dynamical models to measure the mass of the central supermassive black hole to $\sim 5 \times 10^{7}$ $M_{\odot}$.

The molecular barlike structure observed in CO maps taken with the IRAM millimeter interferometer on the Plateau de Bure shows no clear signatures of bar kinematics (Davies et al. 2004) and it does not have a counterpart in highresolution $V$ - or $K$-band images or radio continuum maps analyzed by Genzel et al. (1995), Mulchaey et al. (1997), Lai et al. (1999), Scoville et al. (2000), and Colina et al. (2001; see also Figure 1). Associating the molecular barlike morphology with a non-axisymmetric perturbation raises the problem of locating the position of the intensely star-forming ringlike structure. With its dominant recent burst of star formation, it would be likely placed in, or near, an associated inner resonance radius (e.g., Regan \& Teuben 2003; Piñol-Ferrer et al. 2014; Li et al. 2015).

Given that no significant kinematic characteristics of a barred potential have been found by Davies et al. (2004), it has been suggested that what appears to be a bar is likely a blending of gas clumps. Nevertheless, these authors assumed epicyclic approximation to associate the location of the ringlike structure with the inner Lindblad resonance radius of a primary bar, rotating at a pattern speed of $<80 \mathrm{~km} \mathrm{~s}^{-1} \mathrm{kpc}^{-1}$. Accordingly, 


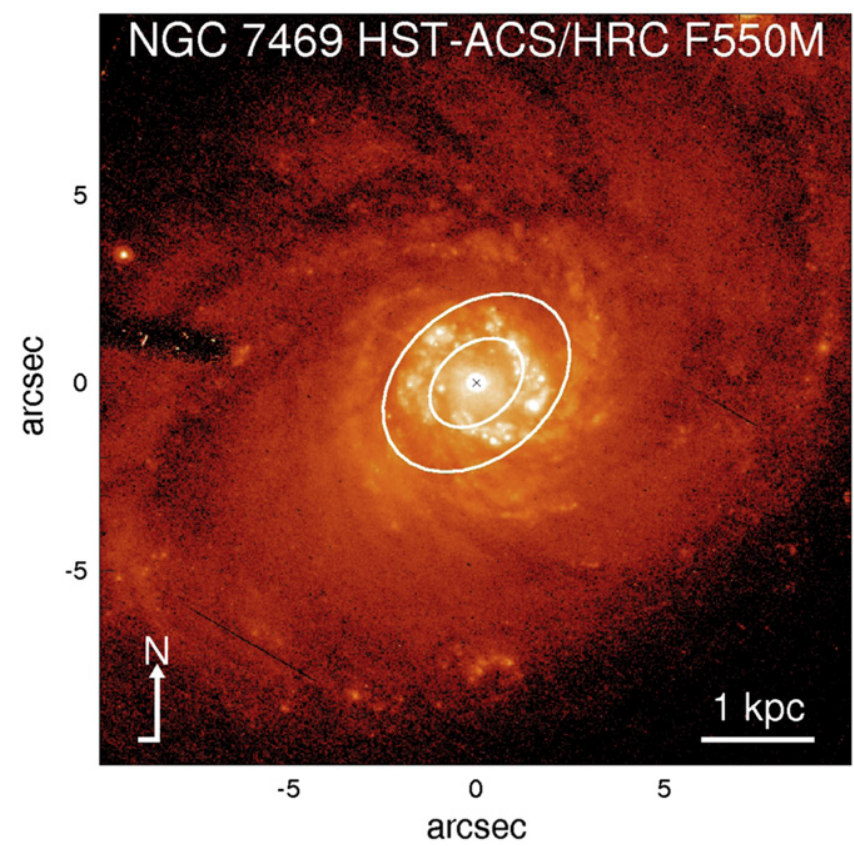

Figure 1. Central region of NGC 7469 with ellipses marking the 500 and $1000 \mathrm{pc}$ boundaries in the plane of the galaxy disk.

the pattern speed for the region inside the ring was estimated to $235 \pm 5 \mathrm{~km} \mathrm{~s}^{-1} \mathrm{kpc}^{-1}$. Congruent with this picture, the starforming regions in NGC 7469 would be supported by its nonaxisymmetric structure(s). However, strong bar/spiral-induced flows would be expected to sustain the large amounts of molecular gas (gas-to-dust ratio $\sim 600$ ) with $\mathrm{HCN} J(4 \rightarrow 3)$ ) CO $J(3 \rightarrow 2) \sim 1$ (Papadopoulos et al. 2012).

While these results provide important clues on the fate of the interstellar gas in NGC 7469, a concise picture for the dynamical effects that trigger the intense star formation in this galaxy is missing. To build a realistic scenario for the relative role of the local and global instabilities that ignite these intense bursts of star formation in the central kiloparsec radius of NGC 7469, we present here a detailed analysis of the distribution and kinematics of the densest phase of the molecular gas in the region of interest. We use the $\mathrm{HCO}^{+}$ $J(4 \rightarrow 3)$ and $\operatorname{HCN} J(4 \rightarrow 3)$ emission lines at high spatial and velocity resolution to derive the instability parameter and the characteristic wavenumbers that describe the local instability scales.

Throughout this work, we assume NGC 7469 to be at an inclination of $45^{\circ}$ and a distance of $68 \mathrm{Mpc}$ (de Vaucouleurs et al. 1991), implying $330 \mathrm{pc} /{ }^{\prime \prime}$, and consistent with the aforementioned studies. The high angular resolution of Atacama Large Millimeter/sub-millimeter Array (ALMA) allows us to characterize the stability of the molecular gas throughout the central kiloparsec region in this prototype luminous infrared galaxy. We show for the first time that it is possible to directly quantify the stability criteria that lead to the star formation in the ringlike structure, accounting for up to two-thirds of the galaxy's bolometric luminosity.

\section{OBSERVATIONS, MAPS, AND PROFILES}

We observed NGC 7469 with ALMA on 2013 November 3 and 4, using 28 antennas in a C32-2 configuration (Proposal ID 2012.1.00165.S, PI: Takuma Izumi). With $18^{\prime \prime}$ primary beam size and $0 . " 5 \times 0$." 4 synthesized beam size, sampled at 0 . 1 pixels $^{-1}$, the ALMA Band 7 receiver was tuned to cover the redshifted lines of $\mathrm{HCN} J(4 \rightarrow 3)\left(\nu_{\text {rest }}=354.505 \mathrm{GHz}\right)$ and $\mathrm{HCO}^{+} J(4 \rightarrow 3)\left(\nu_{\text {rest }}=356.734 \mathrm{GHz}\right)$. The original velocity resolution was $0.43 \mathrm{~km} \mathrm{~s}^{-1}$, but to achieve the amplitude-over-noise level $\geqslant 2$, we binned the data at an effective resolution of $20.2 \mathrm{~km} \mathrm{~s}^{-1}$ (see Izumi et al. 2015a for further details).

To derive the gas distribution and kinematic information, we applied single-Gaussian fits to each individual spectrum in the data cube, keeping only the positive-amplitude fits. Comparison between Gaussian fitting results and conventional moment maps confirm that the line fluxes, line-of-sight velocities, and velocity dispersions $(\sigma)$ as resulting from Gaussian fits are all reliable and fully consistent with the maps presented in Izumi et al. (2015a). The difference is that our fitting scheme allows retrieving maps with a wider spatial coverage and recovering more extended emission, making our maps more suitable for the analysis of the gas kinematics. We further estimated the contribution of the side-lobes to be $<15 \%$ at $R<3^{\prime \prime}$, hence not large enough to distort the derived kinematics (see Figure 2). While the emission line amplitudes slightly vary for the different tracers, we note that their morphology remains unchanged.

Galactocentric flux and $\sigma$ profiles are derived applying robust statistics, ensuring reliable results with up to $50 \%$ tolerance level. Moreover, the above estimated kinematics depend on the implicit assumption that the data comprise a random sample from a normal distribution. Noisy values or regions with a stronger presence of a secondary component are thus accounted for by using robust statistics. Accordingly, we cut each two-dimensional map into projected circular galactocentric rings. At each radius (in the plane of the galaxy), we derive the median value for the flux and $\sigma$, followed by the error in the derived median values as described in Huber \& Ronchetti (2009).

Numerous tests with varying ring widths and ring radii confirm that the exceptional quality and broad dynamical range of the ALMA data allow a stable median and error estimation even when median fluxes and $\sigma$ values are derived at radii a factor of two smaller than the synthesized beam size. The smallest reliable step is thus 0.25 , corresponding to an increment value of $82.5 \mathrm{pc}$, and this will be the step size adopted throughout the analysis presented here. Furthermore, the median velocity error across the field is $5.5 \mathrm{~km} \mathrm{~s}^{-1}$, and the median emission line amplitude error across the field is $0.06 \mathrm{mJy}$ beam $^{-1} \mathrm{~km} \mathrm{~s}^{-1}$. All these parameters remain within the uncertainties both for the $\mathrm{HCN}$ and the $\mathrm{HCO}^{+}$lines.

At these radial steps, we also calculate the rotation curve by assuming that the circular rotation is the dominant kinematic feature and that our measurements refer to positions on a single inclined disk. We then use the method described in, e.g., Schoenmakers et al. (1997), Fathi et al. (2005), and van de Ven \& Fathi (2010) to derive the best representation of the rotation curve. Accordingly, the line-of-sight velocity is written as the harmonic series $k_{0}(R)+\sum_{m=1}^{n} k_{m}(R) \cos \left(m\left[\psi-\psi_{m}(R)\right]\right)$, where $k_{0}$ is the systemic velocity, $k_{1}$ is the best-fit circular velocity, $R$ is the galactocentric radius, $\left.k_{m}\right|_{m>1}$ are higher-order velocity amplitudes, and $\psi_{m}$ are phase shifts (see also van de Ven $\&$ Fathi 2010; and Figure 3 here). Adopting a $45^{\circ}$ inclination, we find that the mean systemic velocity is $4860 \mathrm{~km} \mathrm{~s}^{-1}$ and the kinematic position angle is $132^{\circ}$. Once the rotation curve is 


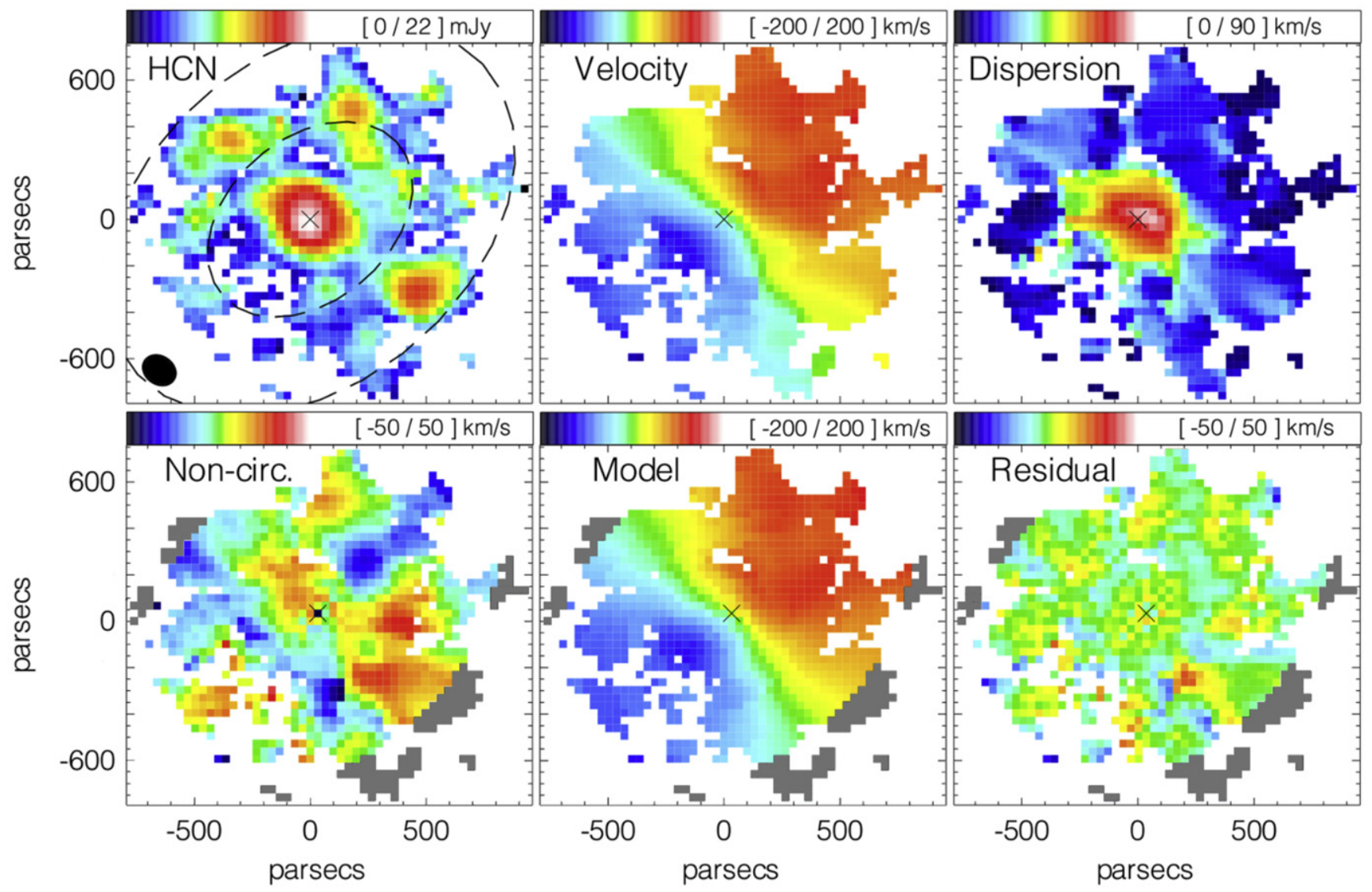

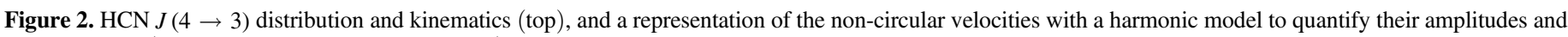

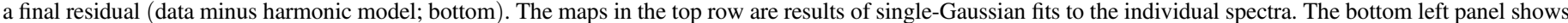

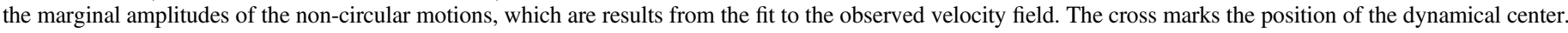

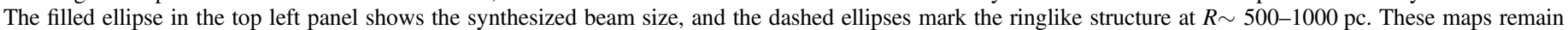
consistent for the $\mathrm{HCO}^{+} J(4 \rightarrow 3)$ tracer, but slightly noisier.

derived at each radius $R$, we calculate the angular frequency $\Omega=k_{1} / R$ and the epicyclic frequency $\kappa=\sqrt{R d \Omega^{2} / d R+4 \Omega^{2}}$ curves (see Figure 3).

\section{RESULTS AND DISCUSSION}

Rotation dominates the kinematics of the disk, and our data show that both the $\mathrm{HCN}$ and the $\mathrm{HCO}^{+}$lines trace the same dynamical entity. Henceforth, we use the $\mathrm{HCN}$, which provides less noisy maps, to derive the dynamical properties of the dense interstellar component, associated with the high-mass starforming gas in this galaxy.

\subsection{Dynamical Mass}

The low central $V / \sigma(\sim 0.5)$ found by Hicks et al. (2009) indicates that the random motions play a significant role in the central parts of NGC 7469. Figure 3 also confirms that the random motions have to be accounted for when deriving the dynamical mass. We assume that the $\mathrm{HCN} \sigma$ rises due to symmetric macroscopic motions, and we calculate the dynamical mass in the central kiloparsec radius applying the equation $M=R\left(k_{1}^{2}+3 \sigma^{2}\right) / G$ (Bender et al. 1992), where $R$ is the radius, $k_{1}$ is the best representation of the rotation curve (as described above), and $G$ is the gravitational constant (see Figure 3 ). The mass curve shown in Figure 3 is in full agreement with the measurements listed in Genzel et al. (1995) and Hicks et al. (2009), and at the ringlike feature, our derived mass can encompass the combined masses for the star-forming regions, as derived by Díaz-Santos et al. (2007).

\subsection{Surface Density of the Dense Gas Phase}

Considering the narrow range of the critical densities and the similarity of the line shapes, it is reasonable to conclude that the $\mathrm{HCN}$ and $\mathrm{HCO}^{+}$are both emitted from the same volume (Wada et al. 2009; Zhang et al. 2014), associated with the dense cores of star-forming giant molecular clouds (Krips et al. 2008; Greve et al. 2009; Andrews \& Thompson 2011). Modeling by Meijerink et al. (2007) and Kazandjian et al. (2012) determined the thermal and chemical balance in similar environments and found that the densities in these regions vary between $10^{4}$ and $10^{6} \mathrm{~cm}^{-3}$. In particular, where $\mathrm{HCN} / \mathrm{HCO}^{+}$ ratio is greater than unity, the most likely number density is $n_{\left(\mathrm{H}_{2}\right)} \sim 10^{5} \mathrm{~cm}^{-3}$. NGC 7469 fulfills this criterion (Izumi et al. 2015a).

In Arp 220-like environments, Greve et al. (2009) found a wide range of kinetic temperatures $T_{k}=10-120 \mathrm{~K}$. Moderately star-forming galaxies exhibit values closer to the lower end of this temperature range (e.g., Gao \& Solomon 2004; Krips et al. 2008; Imanishi et al. 2010); hence, we find it reasonable to assume $T_{k}=25 \mathrm{~K}$ in NGC 7469 . 


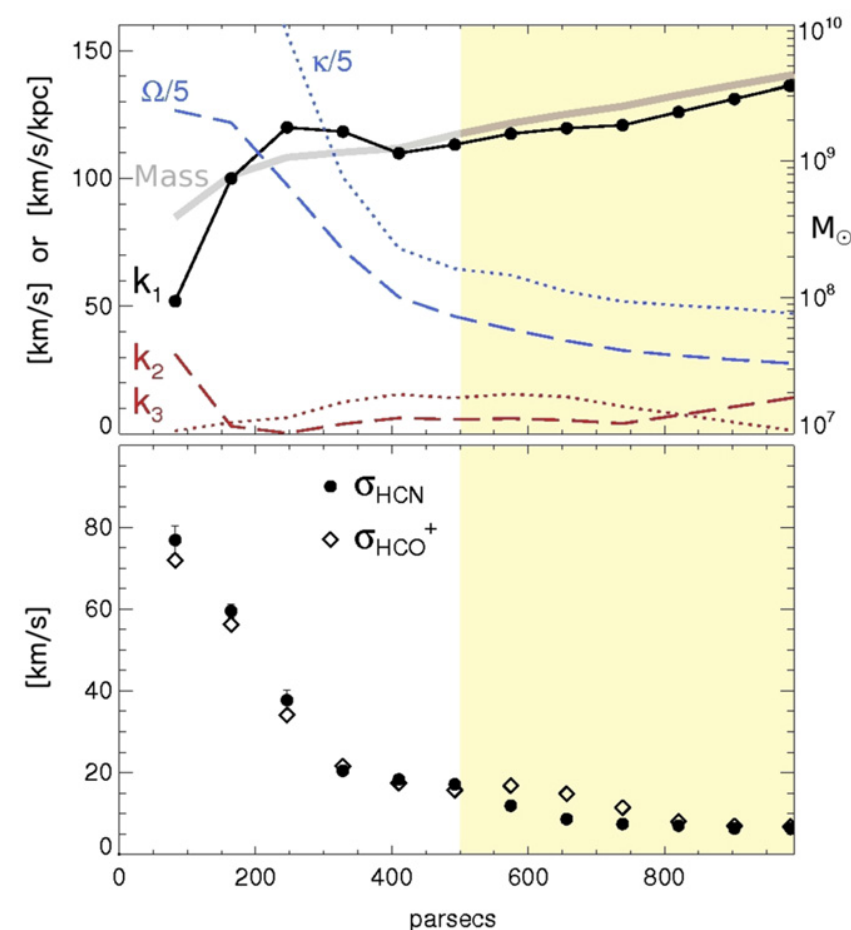

Figure 3. One-dimensional profiles for the best-fit rotation curve $k_{1}$ and corresponding angular frequency $\Omega$ and epicyclic frequency $\kappa$, and the noncircular motions up to and including the third order (top panel), all in the plane of the sky. Both frequency curves are defined in Section 2, and they are here divided by five for illustration purposes. The dynamical mass (thick gray curve) accounts for the rotation curve, the $\mathrm{HCN} \sigma$ values shown in the bottom panel, and the assumed $45^{\circ}$ inclination. The $\mathrm{HCO}^{+} \sigma$ profile is shown as black diamonds. The shaded area marks the ringlike structure.

Using the above density and temperature throughout the entire region studied here, we calculate the conversion factor $X_{\mathrm{HCN}}=2.1 \times \sqrt{n_{\left(\mathrm{H}_{2}\right)}} / T_{b} \sim 27 M_{\odot}\left(\mathrm{K} \mathrm{km} \mathrm{s}^{-1} \mathrm{pc}^{2}\right)^{-1} \quad$ (Krips et al. 2008). Combined with the ratio $\mathrm{HCN} J(4 \rightarrow 3) / \mathrm{HCN}$ $J(1 \rightarrow 0)=0.6$ (Viti et al. 2014), we convert the HCN flux into molecular gas surface density $\Sigma$ (in $M_{\odot} \mathrm{pc}^{-2}$ ). We note that the uncertainties in the density, temperature, and HCN $J(4 \rightarrow 3) / \mathrm{HCN} J(1 \rightarrow 0)$ are large enough to dominate the uncertainties in the $\Sigma$ values illustrated in Figure 4. Both $\Sigma$ and disk stability parameter (as described in Section 3.3) depend highly on the assumed temperature and number density. Decreasing the $\mathrm{HCN} J(4 \rightarrow 3) / \mathrm{HCN} J(1 \rightarrow 0)$ will linearly increase the density values, while increasing the temperature will decrease the conversion factor.

Given that the active galactic nucleus in NGC 7469 only influences the inner $<40 \mathrm{pcs}$ (Viti et al. 2014; Izumi et al. 2015b), our $\Sigma$ curve is not affected by the corresponding radiation field. We further note that our measurements are prone to beam dilution up to a factor of five (e.g., Sakamoto et al. 2011) and that this effect would translate into a similar level of underestimation of the $\Sigma$. Hence, we provide a rough estimate of the uncertainties for the $\Sigma$ and subsequent stability parameter, using a factor of five as the upper value, and a factor of two higher kinetic temperature (e.g., Scoville et al. 2015) as the lower $\Sigma$ limit (Figure 4).

\subsection{Disk Stability Analysis}

To analyze the disk stability in the central $R \sim 1 \mathrm{kpc}$ of NGC 7469, we assume that the velocity dispersion is isotropic

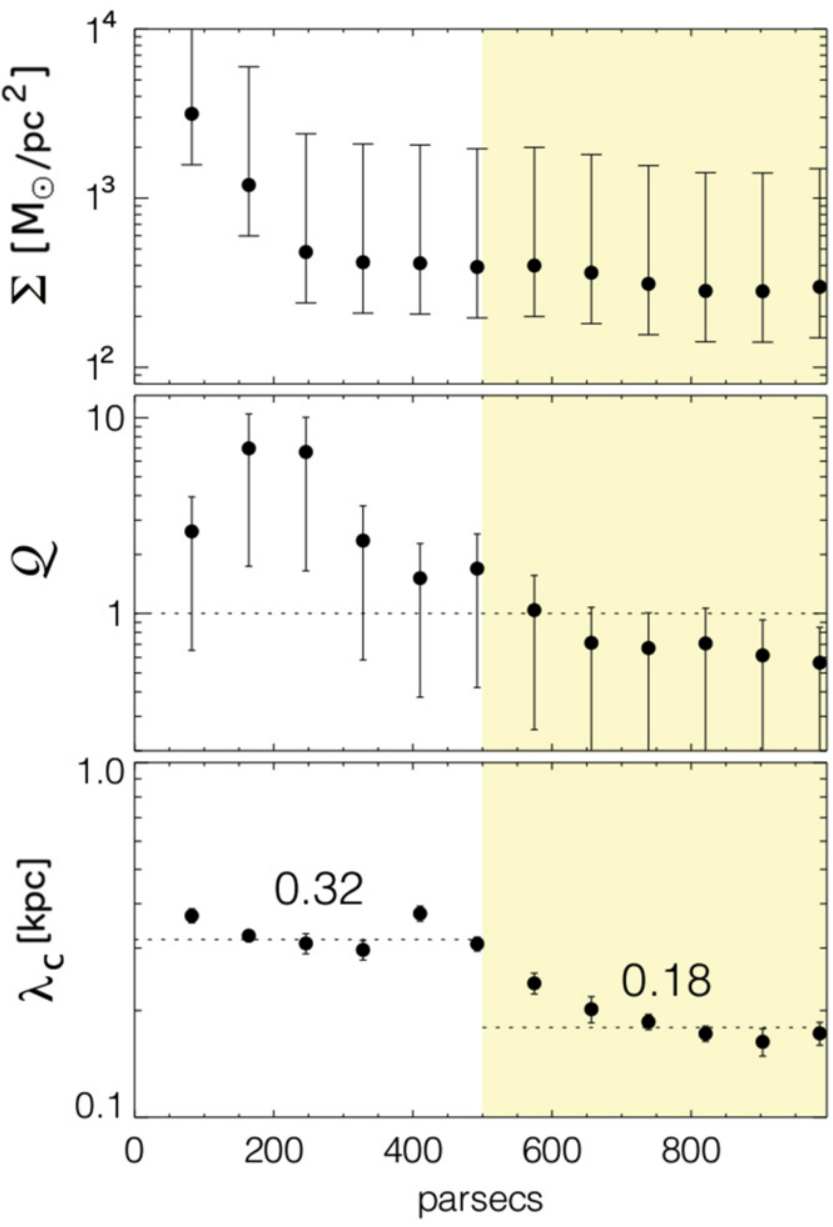

Figure 4. HCN surface density $\Sigma$, stability parameter $\mathcal{Q}$, and instability scale $\lambda_{c}$ (top, middle, and bottom panel, respectively). The two dashed lines in the bottom panel mark the median value within each region, as indicated. The shaded area marks the ringlike structure of intense star formation. The uncertainties in $\Sigma$ (as described in Section 3.2) are taken into account when deriving $\mathcal{Q}$ (see also Section 3.3).

and we derive the Toomre-like stability parameter $\mathcal{Q}$ for the $\mathrm{HCN}$, also taking into account the disk thickness (Romeo \& Wiegert 2011; Romeo \& Falstad 2013). Accordingly, $\mathcal{Q}=3 \kappa \sigma / 2 \pi G \Sigma$, where all the parameters are described above. Romeo \& Wiegert (2011) have further shown that the dispersion relation for the stability parameter $\mathcal{Q}$ converges to the Jeans dispersion relation. The middle panel of Figure 4 illustrates that while the stability parameter $\mathcal{Q}$ is consistently above unity where no star formation is observed, this parameter decreases below unity at the region of the ringlike structure in NGC 7469. The corresponding error bars account for the uncertainties in the surface density $\Sigma$ and the errors in the derived median $\sigma$ values.

This is a strong result since it shows that even the densest gas, which is likely sunk to the disk mid-plane (McKee \& Ostriker 2007) and least prone to perturbations, is unstable in the ringlike structure. The instability gives rise to the intense formation of the high-mass young stars that have been reported in the literature (Díaz-Santos et al. 2007). Another interesting point here is that the $\operatorname{HCN} \mathcal{Q}$ highlights the role of local gravitational instabilities that lead to the intense star formation in this galaxy. Romeo \& Fathi (2015) presented a detailed discussion on the physical phenomena that could contribute to 
the high $\mathcal{Q}$ at small radii. Moreover, while gas turbulence alters the condition for star-gas decoupling and increases the least stable wavelength, it hardly modifies the Q parameter at scales larger than about $100 \mathrm{pc}$ (Hoffmann \& Romeo 2012; Agertz et al. 2015). Given the metallicity of the central kiloparsec radius of NGC 7469, it is possible that CO-dark molecular gas, anisotropy of the gas velocity dispersion, and non-axisymmetric perturbations all contribute to the disk stability at $R \lesssim 500$ pc region, by up to $50 \%$ or so. However, outside this radius, the low-velocity dispersion of the HCN suggests that the contribution of the CO-dark gas and anisotropy may be marginal (Romeo \& Fathi 2015). Furthermore, Sani et al. (2012) found that the Toomre parameter traced by HCN is above unity at the center of other Seyfert galaxies.

The prescription of Romeo \& Falstad (2013) further allows measuring the characteristic instability scale $\lambda_{c}$, i.e., the physical scales at which a realistically thick galactic disk becomes locally unstable as $\mathcal{Q}$ drops below unity. It is defined as $\lambda_{c}=2 \pi \sigma / \kappa$ and deriving the scales for local perturbations does not exclude the role of global perturbation in reshaping the disk in NGC 7469. Indeed, a locally stable disk can still be globally unstable to bi-symmetric gravitational perturbations. Moreover, the dynamics and evolution of disk structures depend critically on the radial profile of the $\mathcal{Q}$ stability parameter (Romeo \& Falstad 2013 and references therein).

The lower panel in Figure 4 shows a marked difference between $\lambda_{c}$ interior to and on the ringlike structure in NGC 7469. It is striking that in this region our measured $\sim 180 \mathrm{pc}$ scale (median $\lambda_{c}=0.18 \mathrm{kpc}$ ) is fully comparable to the measured sizes for the star-forming complexes. The derived local instability scale for the densest gas is further supported by the observed young $(5-6 \mathrm{Myr})$ and highly obscured $\left(A_{V} \sim 10\right.$ mag) population of stars that coexist with an intermediate age (15-35 Myr) less extincted population (Díaz-Santos et al. 2007). The young and massive population accounts for approximately one-third of the total stellar mass and up to twothirds of the infrared luminosity in the ringlike structure (DíazSantos et al. 2007).

\section{CONCLUSIONS}

Studies of star formation in disks suggest that massive stars are predominantly formed in the densest cores of giant molecular clouds (e.g., Krumholz 2014); hence, it is commonly assumed that $\mathrm{HCN}$ and $\mathrm{HCO}^{+}$trace these dense cores $(\mathrm{Wu}$ et al. 2005; Lada et al. 2012). Due to the high critical densities, a tighter correlation is found between the intensities of these molecules and the far-infrared emission (Gao \& Solomon 2004). This correlation further extends to dense cores undergoing high-mass star formation (Lada et al. 2012; Zhang et al. 2014).

The galactic-scale intense star formation in NGC 7469 is likely due to the combination of a past interaction with the neighboring IC 5283 and the presence of its bar or spiral arms. However, the expected non-circular motions are not detected in our ALMA kinematic maps for this galaxy (cf. Fathi et al. 2005, 2006, 2013). Our ALMA observations of HCN $J(4 \rightarrow 3)$ and $\mathrm{HCO}^{+} J(4 \rightarrow 3)$ lines reveal a number of interesting clues on how local gravitational instabilities help forming stars in this galaxy.

We derive the radial profiles for the disk stability parameter $\mathcal{Q}$ and the characteristic instability scale $\lambda_{c}$ in NGC 7469. We find signatures of disk instability on the ringlike structure, inside which the disk is stable against star formation. At the highly star-forming ringlike structure, the median local instability scale is $\sim 180 \mathrm{pc}$, in full agreement with the sizes of individual star-forming complexes seen in Hubble Space Telescope images. Our stability analysis accounts for a disk structure with finite thickness, and as discussed in Romeo \& Fathi (2015) and Agertz et al. (2015), turbulence could alter the condition for star-gas decoupling and increases the least stable wavelength, but hardly modifies the stability parameter $\mathcal{Q}$ at scales larger than about $100 \mathrm{pc}$.

Our robust results further show that short-wavelength approximation is satisfied in the region studied here and that the ringlike structure could in essence be only a tightening of the galactic-scale non-axisymmetric spiral arms. It will be interesting to apply this analysis to the warmer, less dense, and more diffuse CO gas; however, as it was pointed out by Romeo \& Falstad (2013), such a comparison must be based on identical data quality and resolution. At the moment we have no $\mathrm{CO}$ data at hand that match the resolution, sensitivity, and spatial coverage of the ALMA observations analyzed here.

We thank the anonymous referee for insightful comments that helped improve our manuscript. This paper makes use of the following ALMA data: ADS/JAO.ALMA\#2012.1.00165.S. ALMA is a partnership of ESO (representing its member states), NSF (USA) and NINS (Japan), together with NRC (Canada), NSC and ASIAA (Taiwan), and KASI (Republic of Korea), in cooperation with the Republic of Chile. The Joint ALMA Observatory is operated by ESO, AUI/NRAO and NAOJ. S.M. is supported by MoST 103-2112-M-001-032MY3. K.F. acknowledges the hospitality of ESO in Garching, where part of this work was carried out.

\section{REFERENCES}

Agertz, O., Romeo, A. B., \& Grisdale, K. 2015, MNRAS, 449, 2156 Andrews, B. H., \& Thompson, T. A. 2011, ApJ, 727, 97

Bender, R., Burstein, D., \& Faber, S. M. 1992, ApJ, 399, 462

Bolatto, A. D., Wolfire, M., \& Leroy, A. K. 2013, ARA\&A, 51, 207 Colina, L., Alberdi, A., Torrelles, J. M., Panagia, N., \& Wilson, A. S. 2001, ApJL, 553, L19

Davies, R., Tacconi, L., \& Genzel, R. 2004, ApJ, 602, 148

Davies, R., Sternberg, A., Lehnert, M., \& Tacconi-Garman, L. 2005, ApJ, 633, 105

de Vaucouleurs, G., de Vaucouleurs, A., Corwin, H. G., et al. 1991, Third Reference Catalogue of Bright Galaxies (New York: Springer)

Díaz-Santos, T., Alonso-Herrero, A., Colina, L., Ryder, S. D., \& Knapen, J. H. 2007, ApJ, 661, 149

Fathi, K., van de Ven, G., Peletier, R. F., et al. 2005, MNRAS, 364, 773

Fathi, K., Storchi-Bergmann, T., Riffel, R., et al. 2006, ApJL, 641, L25

Fathi, K., Lundgren, A. A., Kohno, K., et al. 2013, ApJL, 770, L27

Gao, Y., \& Solomon, P. M. 2004, ApJ, 606, 271

Genzel, R., Weitzel, L. E., Tacconi-Garman, L., et al. 1995, ApJ, 444, 129

Greve, T. R., Papadopoulos, P. P., Gao, Y., \& Radford, S. J. E. 2009, ApJ, 692, 1432

Hicks, E. K. S., \& Malkan, M. A. 2008, ApJS, 174, 31

Hicks, E. K. S., Davies, R. I., Malkan, M. A., et al. 2009, ApJ, 696, 448

Hoffmann, V., \& Romeo, A. B. 2012, MNRAS, 425, 1511

Huber, P. J., \& Ronchetti, E. M. 2009, Robust Statistics (Hoboken, NJ: John Wiley \& Sons)

Imanishi, M., Nakanishi, K., Yamada, M., Tamura, Y., \& Kohno, K. 2010, PASJ, 62, 201

Izumi, T., Kohno, K., Aalto, S., et al. 2015a, ApJ, submitted

Izumi, T., Kohno, K., Aalto, S., et al. 2015b, ApJ, submitted

Kazandjian, M. V., Meijerink, R., Pelupessy, I., Israel, F. P., \& Spaans, M. 2012, A\&A, 542, 65

Krips, M., Neri, R., García-Burillo, S., et al. 2008, ApJ, 677, 262

Krumholz, M. R. 2014, ASSL, 412, 43

Lada, C. J., Forbrich, J., Lombardi, M., \& Alvez, J. F. 2012, ApJ, 669, 289 
Lai, O., Rouan, D., \& Alloin, D. 1999, in Astronomy with Adaptive Optics, ed. Bonaccini (Garching: ESO), 555

Li, Z., Shen, J., \& Woong-Tae, K. 2015, ApJ, submitted (arXiv:1503.02594) Mauder, W., Weigelt, G., Appenzeller, I., \& Wagner, S. J. 1994, A\&A, 285, 44

McKee, C. F., \& Ostriker, E. C. 2007, ARA\&A, 45, 565

Meijerink, R., Spaans, M., \& Israel, F. P. 2007, A\&A, 461, 793

Meixner, M., Puchalsky, R., Blitz, L., Wright, M., \& Heckman, T. 1990, ApJ, 354,158

Mulchaey, J. S., Regan, M. W., \& Kundu, A. 1997, ApJS, 110, 299

Papadopoulos, P. P., van der Werf, P. P., Xilouris, E. M., et al. 2012, MNRAS, 426, 2601

Piñol-Ferrer, N., Fathi, K., Carignan, C., et al. 2014, MNRAS, 438, 971

Regan, M. W., \& Teuben, P. 2003, ApJ, 582, 723

Romeo, A. B., \& Falstad, N. 2013, MNRAS, 433, 1389
Romeo, A. B., \& Fathi, K. 2015, MNRAS, in press (arXiv:1503.01326)

Romeo, A. B., \& Wiegert, J. 2011, MNRAS, 416, 1191

Sakamoto, K., Map, R.-Q., Matsushita, S., et al. 2011, ApJ, 735, 19

Salamanca, I., Alloin, D., \& Pelat, D. 1995, A\&ASS, 111, 283

Sani, E., Davies, R. I., Sternberg, A., et al. 2012, MNRAS, 424, 1963

Schoenmakers, R. H., Franx, M., \& de Zeeuw, P. T. 1997, MNRAS, 292, 349

Scoville, N., Evans, A. S., Thompson, R., et al. 2000, AJ, 119, 991

Scoville, N., Faisst, A., Capak, P., et al. 2015, ApJ, 800, 70

van de Ven, G., \& Fathi, K. 2010, ApJ, 723, 767

Viti, S., García-Burillo, S., Fuente, A., et al. 2014, A\&A, 570, 28

Wada, K., Papadopoulos, P. P., \& Spaans, M. 2009, ApJ, 702, 63

Wu, J., Evans, N. J. II, Gao, Y., et al. 2005, ApJL, 635, L173

Zhang, Z., Gao, Y., Henkel, C., et al. 2014, ApJ, 784, 31 